\title{
Diagnostic Dilemma of an Unusual Pelvic Mass in a Young Girl
}

\author{
Rajbhandari S, ${ }^{1}$ Shrestha B, ${ }^{1}$ Karki A' \\ 'Kathmandu Model Hospital, Exhibition Road, Kathmandu, Nepal.
}

\begin{abstract}
Dysgerminoma of ovary is the most common germ cell tumor, accounting for $50 \%$ of all germ cell tumor cases. About $20 \%$ of cases are diagnosed during pregnancy, and $80 \%$ occur in women under 30. It is rare to find both ovaries to be involved in germ cell tumors. The prognosis of patients with malignant germ cell has improved significantly over the last two decades after the introduction of chemotherapy specially cisplatin. The only exceptions are stage 1, grade1, immature teratoma and stage 1A dysgerminoima who are followed up after surgery without adjuvant chemotherapy. Normal ovarian functions and fertility can be retained in most patients by following the conservative surgery.
\end{abstract}

Key words: Conservative surgery, dysgerminoma, germ cell tumor.

\section{INTRODUCTION}

The dysgerminoma represents the ovarian counterpart of the testicular seminoma. These two tumors are histologically identical and the term germinoma has been proposed for both. It usually occurs in normally developed females but is the most frequent ovarian malignant tumor found in dysgenetic females, testicular feminization, and hermaphrodites and ambiguous sex. Dysgerminomas tend to be large, solid and bosselated with a smooth surface. The cut surface is soft, fleshy and bulging with a homogeneous pink-tan color. Ultrasonography (USG) and Computerized tomography scan is vital for diagnosis. We present a case of dysgerminoma which took a long time to come to final diagnosis

\section{CASE REPORT}

A young unmarried girl of 24 years came to Kathmandu Model Hospital with discomfort in the lower abdomen, which was not associated with pain. There was no history of fever, loss of weight or appetite, no sexual activity and no urinary or bowel movement problems. Menstrual cycle was regular, no dysmenorrhoea and normal flow. Family history of mother's lung cancer existed, which was diagnosed at a late stage. The girl was lean and thin with no other positive findings except irregular very firm, linear hard mass just above inguinal ligament; non-tender, non mobile and secondary sexual characters were normal. Complete blood picture was within normal limit.

Ultrasonography (USG) report showed large pelvic mass origin was probably ovarian, tubes and ovary on the other side was found to be normal. Irregular inhomogeneous, hypoechoeic structure in the pelvic region, which was more towards the right side, measured $11.1 \times 7.4 \times 8.7 \mathrm{~cm}$ and the uterus, was normal in size. There was no ascites or any abnormalities of other abdominal organs.

The dilemma in this case was the mass felt irregular bony and it was difficult to make a provisional diagnosis of the ovarian origin. Hence complete investigation was planned and opinions were sought from general surgeon, physician and neurosurgeon.

\author{
Correspondence: \\ Dr. Swaraj Rajbhandari \\ Kathmandu Model Hospital \\ Kathmandu, Nepal. \\ Email: swarajr@hotmail.com
}


Initially surgeons thought of possible tubercular mass. Physician's opinion was Koch's abdomen as Mountoux test was $32 \mathrm{~mm}$ after 48 hours. She was put on antitubercular treatment for a month but she discontinued due to its side effects. Neurosurgeon's opinion was that the feel of the mass could be chondroma.

Computerized Tomography scans showed quite large ovarian tumor on the right side, and $10.4 \times 8.9 \times 7 \mathrm{cms}$. Serum CA-125 was found to be $15 \mathrm{lU} / \mathrm{ml}$ during the same visit. Repeat USG after four weeks large mass about $11 \times 63 \times 82 \mathrm{~cm}$ seen on the anterior to the uterus with nodular and sharp margins. The left ovary was normal and the right ovary was not identified. USG impression was a large pedunculated fibroid with minimal ascites. Follow up visit after five months almost the same findings as stated above so counseled for diagnostic laparotomy but the patient refused.

Two weeks later after her last follow up she agreed to undergo surgery as she could palpate the mass herself. During this visit, abdominal finding was completely different. This time a mobile mass of about 14 weeks size firm in consistency and non-tender was found.

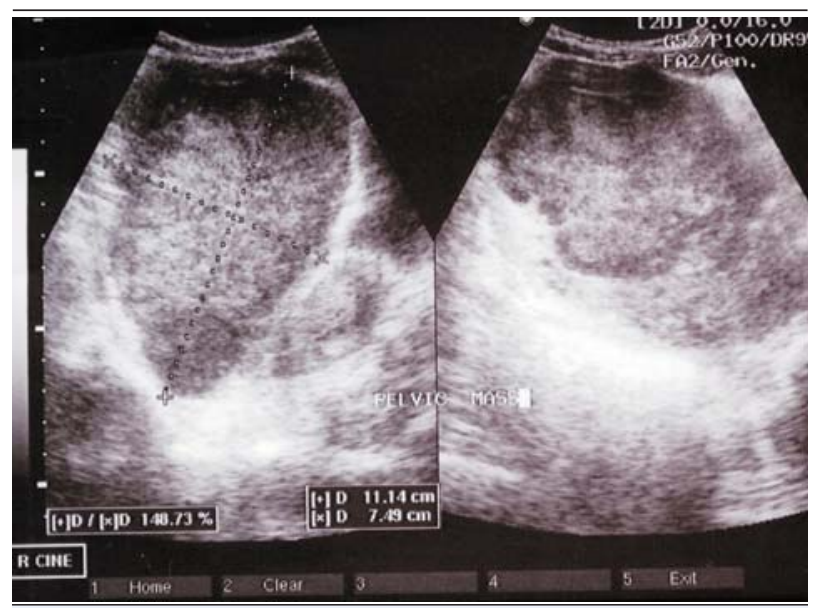

Figure 1. USG showing pelvic mass.

On laparotomy no adhesions, uterus and left ovary was normal, right ovarian mass of about $12 \times 9 \mathrm{~cm}$, brain like lobulated firm mass tube was adherent, uterus and contralateral ovary and tube was normal, pelvic and para-aortic nodes were carefully examined and found to be not enlarged. There was intussusception of ileum which was cleared by milking. According to FIGO staging of Ovarian Germ cell tumours: Stage 1: tumor limited to one ovary, no ascites and intact capsule. Postoperative period was uneventful. She was discharged on the fifth postoperative day. Histopathology report was dysgerminoma of right Ovary (Figure 1-3).

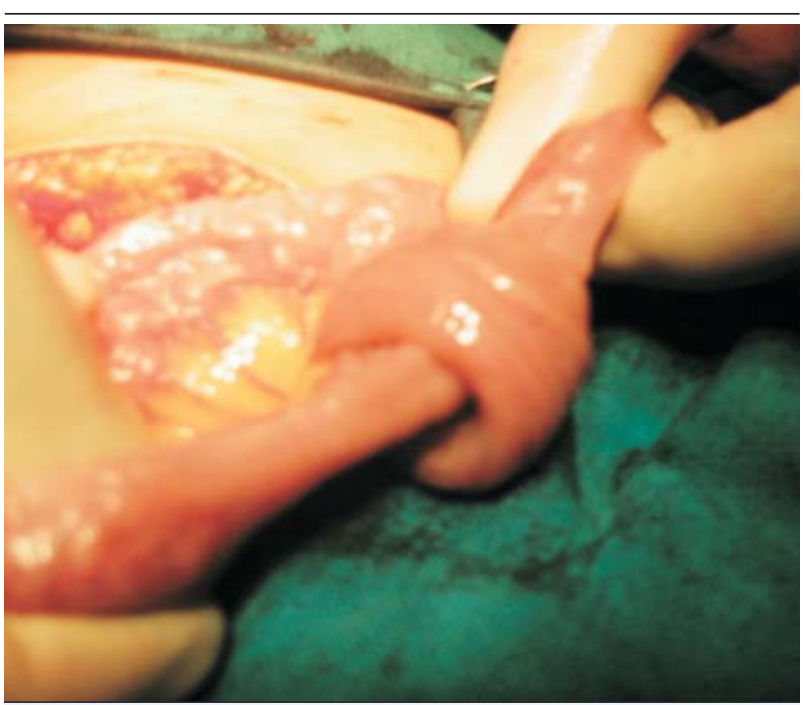

Figure 2. Intussusception on laparotomy.

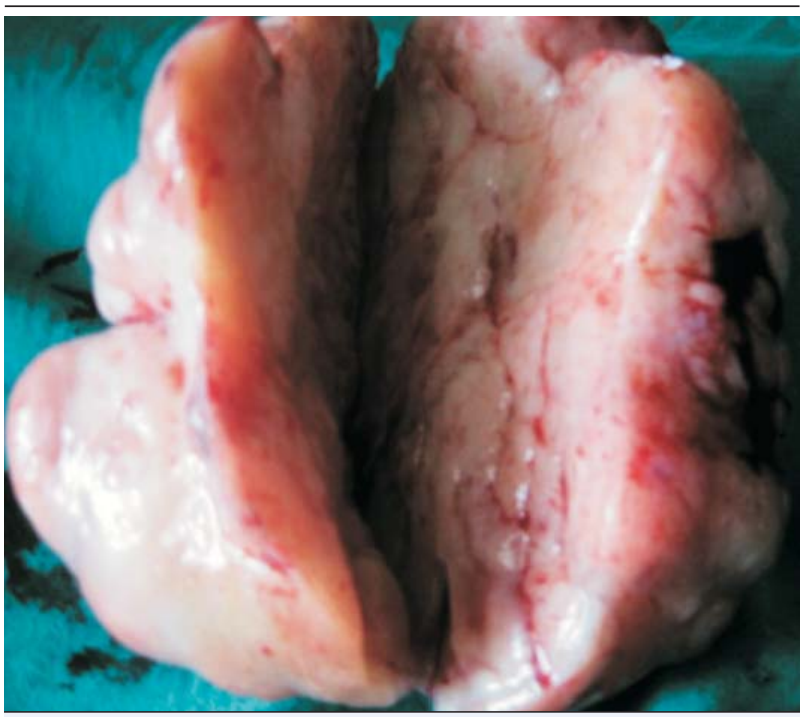

Figure 3. Cut section of the tumor.

\section{DISCUSSIONS}

Germ cell tumors of the ovary account for less than $5 \%$ of ovarian cancers. The median age of malignant germ cell tumor is $6-14$ years and the range is $6-46$ years. ${ }^{1}$ These are found in the second decades of life and frequently diagnosed by a palpable mass associated with pain. Recent development in chemotherapy has dramatically changed the prognosis for many patients who develop the more aggressive type of germ cell tumor.

Ultrasound is the first investigation to get the clue. But clear and accurate sonographic assessment is still a problem as two different diagnoses were given in this case. According to Mainz where 10 sonographic parameters are assessed and scored on a scale of 0-2, 
a score of less than 9 is rated as benign, the sensitivity of this scoring is $96 \%$ and specificity is $80.7 \%$, but the positive predictive value was only $47 \% .{ }^{7}$ The conventional USG is not diagnostic. Hence Magnetic Resonance Image (MRI) is called for to determine the site of origin .Contrast MRI can distinguish between benign and malignant masses with accuracy of $86-95 \% .^{2}$ Computerized Tomography scan although CT scan is the most common imaging modality be used to stage ovarian tumor, MRI has been shown to be equally accurate. ${ }^{8}$

The development of effective combination chemotherapy for young women with malignant ovarian germ cell tumors have been one of the true success story in medicine. Surgery continues to have a pivotal role in the management of all patients with an ovarian tumor. Many germ cell tumors posses the unique property of producing biologic markers that are detected in serum. The development of specific and sensitive radioimmunoassay technique measure Beta HGC and Alpha-fetoprotein (AFP) led to dramatic improvement in monitoring patients with these tumors. Dysgerminoma is commonly devoid of hormonal product small percentage of tumors produce low levels of HCG. A third tumor marker is lactic dehyrdogenase (LDH) and is frequently seen in-patients with dysgerminoma. The level of CA-125 is also elevated in some patients with germ cell tumor but this is also non-specific. Fine needle aspiration biopsy is not so much recommended due to risk of dissemination and also the sensitivity is only $25 \%$ and although specificity being $90 \% .^{10}$

Surgical staging is essential for determining the extent of disease. ${ }^{4}$ For dysgerminoma confined to ovary, or ovaries, no ascites, intact capsule, tumor less than 10 $\mathrm{cm}$ in size with an intact capsule unattached to other organs and without ascites, the 10 year survival rate following conservative surgery was $88.6 \%$ in a series and number of patients had one or more successful pregnancies following unilateral salpingo-oopherectomy. ${ }^{9}$ Conservative management is suggested for the dysgerminoma of stage 1 with proper follow up.

About $15-25 \%$ will recur, but can be treated successfully with combined chemotherapy at the time of recurrence with a high likelihood of cure. Incompletely staged patients or with higher stage tumours probably should receive adjuvant treatment whether adjuvant chemotherapy is recommended or not depends upon on the histologically defined tumor. ${ }^{6}$
For well staged stage 1 patients with dysgerminoma and low grade immature tumor careful observation and follow up are sufficient. Long term survival in patients with stage 1 dysgerminoma who receive no adjuvant therapy is more than $90 \%$. Similarly low grade stage 1 teratoma also have low rate of recurrences. However, high grade immature teratoma should receive 3 or 4 cycles of Bleomycin, Etoposide and Cisplatin (BEP) given every 21 days. In contrast to epithelial ovarian cancer, women with advanced germ cell tumor can often be cured. The prognosis is still good with advanced disease with cure rate of $60-80 \%$.

Recurrence occurs usually within two years of initial therapy. Thus proper follow up every three months in first two years is a must even after completing therapy. Dysgerminoma and immature low rate teratoma can be treated with BEP for recurrence. ${ }^{3}$

Despite the remarkable radio sensitivity of dysgerminoma radiotherapy is rarely performed now days, since chemotherapy is more effective, less toxic and permits preservation of gonadal function. For girls and young women who were successfully treated questions regarding their ability to conceive and carry a pregnancy in future is important. ${ }^{5}$ None of the recent articles reported an increase in the birth defects or miscarriage even when treated with chemotherapy. However, judicious use of surgery followed by chemotherapy will cure majority of patients with ovarian germ cell tumor.

\section{CONCLUSION}

There was a diagnostic dilemma for about two months in this case. It took about nine months to operate; however the delay in surgery was patient's choice. Finally diagnostic laparotomy was done, with removal of mass, unilateral salpingoopherectomy conserving the uterus and opposite ovary, as suggested for the dysgerminoma of stage 1 without any ascites. This case was thoroughly discussed with oncologist and decided to do close followup. Tumor markers during follow-up visits were within the limit like serum beta HCG $(1 \mathrm{mlU} / \mathrm{ml}) /$ and alpha fetoprotein $3.9 \mathrm{ng} / \mathrm{ml}$.

\section{ACKNOWLEDGEMENTS}

We thank Prof. Sanu Maiya Dali (Nepal Medical College) and Dr. Ganesh Dangal (Kathmandu Model Hospital) for reviewing and giving valuable suggestions to this manuscript. Authors would like to thank Ms. Suzu Khadka for formatting the manuscript. 


\section{REFERENCES}

1. Dark GG, Bower M, Newlands ES, et al. Surveillance policy for stage 1 ovarian germ cell tumors. J Clin Oncol 1997;15(2):620-4.

2. Grab D, Flock F, Stohr I, Nussie K, et al. Classification of asymptomatic adnexal masses by ultrasound, magnetic resonance imaging, and positron emission tomography. Gynecol Oncol 2000;77(3):454-9.

3. Hassan E, Creastas G, Deligerologou E, et al. Ovarian tumours during childhood and adolescence: a clininicopathological study. Euro J Gynecol Oncology 1999;20(2):124-6.

4. Higgins RV, Matkins JF, Marroum MC. Comparison of fine needle aspiration cytologic findings of ovarian cysts with histologic findings. Am J Obstet Gynecol 1999;180(3):550-3.

5. Kozlowski KJ. Ovarian masses. Adolesc Med $1999 ; 10(2): 337-50$.

6. Kurman RJ, Noris HJ. Malignant germ cell tumors of the ovary
Human Patholo 1977;8(5):551-64.

7. Mainz E, Weber G, Bahaman F, et al. A new sonomorphologic scoring system (Mainz score) for the assessment of tumours using transvaginal sonography. Part 1: A comparison between the scoring system and the assessment by an experienced sonographer. Ultraschaill Med 1998;19(3):99-107.

8. Woodward PJ, Hosseinzadeh K, Saenger JS. From the archives of the AFIP: radiologic staging of ovarian carcinoma with pathologic correlation. Radiographics 2004 Jan-Feb;24(1):22546.

9. Thomas GM, Dembo AJ, Hacker NF, et al. Current therapy for dysgerminoma of the Ovary. Obstet Gynecology 1987;70(2):268-75.

10. Usbututn A, Altinok G, Kucukali T. The value of intraoperative consultaion (frozen section) in the diagnosis of ovarian neoplasm. Acta Obstet Gynecol Scand 1998;77(10):1013-6. 